\title{
PROPAGATION OF ROSSBY WAVES IN STRATIFIED SHEAR FLOWS
}

\section{PALANI G. KANDASWAMY and B. TAMIL SELVI}

Department of Mathematics Bharathiar University Coimbatore 641046 , INDIA

and

\section{LOKENATH DEBNATH}

Department of Mathematics University of Central Florida Orlando, Florida 32816, U.S.A.

(Received on November 5, 1987 and in revised form March 5, 1988)

ABSTRACT. A study is made of the propagation of Rossby waves in a stably stratified shear flows. The wave equation for the Rossby waves is derived in an isothermal atmosphere on a beta plane in the presence of a latitudinally sheared zonal flow. It is shown that the wave equation is singular at five critical levels, but the wave absorption takes place only at the two levels where the local relative frequency equals in magnitude to the Brunt Vaisala frequency. This analysis also reveals that these two levels exhibit valve effect by allowing the waves to penetrate them from one side only. The absorption coefficient $\exp (2 \pi \mu)$ is determined at these levels. Both the group velocity approach and single wave treatment are employed for the investigation of the problem.

1980 AMS Subject Classification Codes: 76C20, 76V05

Key Words and Phrases: Rossby Waves, critical levels, singularities of the wave equation, wave action flux, and valve effect.

1. INTRODUCTION.

Longuet-Higgins [1-2] used the Rossby $\beta$-plane approximation to investigate the propagation of planetary waves on a rotating sphere. He also studied the characteristic feature of westward propagation of these waves in an unbounded ocean, and also established the existence of a cut-off frequency due to tidal effects above which these waves cease to propagate. Subsequently, Debnath [3] has also investigated the long period response of an unbounded $\beta-p l a n e$ ocean with bottom friction to initial disturbances of wind stress applied at the free surface of the ocean. He also studied the free and forced barotropic motions produced by stationary or travelling atmospheric winds. 
In atmospheric studies, there has been considerable attention to the vertical propagation of Rossby waves, including the interaction between Rossby waves and zonal currents. In a classical paper by Charney and Drazin [4], it was shown that effect on the vertical propagation of Rossby waves of zonal flows in the Earth's atmosphere was to make the dominant planetary waves evanescent in the vertical direction, so that the winds simulate a quasi-solid boundary which prevents the vertical propagation of the large flux of wave energy from the lower to the upper atmosphere. Subsequently, Dickinson [5] studied the behaviour of waves at a critical latitude where the speed of the zonal flow matches with the wind speed in the case of Rossby waves. He has also pointed out the existence of critical latitude which occurs at the intrinsic frequency $\Omega_{d}=0$, where the northward transfer of zonal momentum experiences a finite jump. Later on, Lindzen [6] has shown that a transfer of wave energy and momentum to the wind may take place at such latitudes in a manner which is somewhat similar to the critical layers for gravity waves in a vertically stratified shear flow studied by Booker and Bretherton [7]. It was shown by Brooker and Bretherton that if the component in the direction of the streaming of the phase velocity of a gravity wave matches the flow speed at some point, a critical layer develops and most of the wave energy is absorbed into the mean flow for Richardson numbers of order one or more. It should be pointed out that a critical level is the level at which fluid velocity becomes equal to the horizontal phase velocity of the wave. The wave equation is singular at this level. In their study of a Boussinesq inviscid adiabatic fluid in a non-rotating system, Booker and Bretherton concluded that the waves are attenuated as they pass through the critical level when the Richardson number is greater than $1 / 4$.

Recently, Mekki and McKenzie [8] used the Rossby B-plane approximation to study the propagation of atmospheric Rossby gravity waves in latitudinally sheared zonal flows. They derived a general wave equation for an isothermal atmosphere under the Boussinesq approximation with $\Omega_{d}^{2}<<f^{2}$ and $\Omega_{d}^{2}<<N^{2}$ where the intrinsic frequency $\Omega_{d}=k U-\omega, \omega$ is the frequency of the wave, $k$ is the zonal wavenumber and $U$ is the zonal flow speed, $f$ is the Coriolis parameter and $N$ is the Brunt-Vaisala frequency. They described a wide class of possible wave motions, in the presence of a background zonal flow, ranging from high frequency acoustic-gravity-inertial waves to lowfrequency Rossby waves. Special attention has been given to the propagation properties of Rossby waves in various kinds of latitudinally sheared zonal flows which occur at different heights and seasons in the Earth's atmosphere.

In spite of the above works, relatively less or no attention has been given to the existence and properties of critical levels associated with Rossby waves in an isothermal atmosphere on a $\beta$-plane approximation in the presence of a latitudinally sheared zonal flow. The wave equation related to this problem is derived to investigate its singular behaviour. It is shown that this equation is singular at five critical levels, but the wave absorption takes place only at the two levels where the local relative frequency, $\Omega_{d}$, equals in magnitude to the Brunt-Vaisala frequency. Special attention is given to the conditions for the transmission and capture of the waves at the critical levels. 


\section{BASIC EQUATIONS OF MOTION AND EQUILIBRIUM CONFIGURATION.}

The equations of momentum, continuity, and energy for an inviscid fluid rotating with angular velocity $\underline{\Omega}$ under the gravitational acceleration $\underline{g}$ are given by (see Greenspan [9])

$$
\begin{gathered}
\rho\left[\frac{\partial \underline{u}}{\partial t}+(\underline{u} \cdot \nabla) \underline{u}+2 \underline{\Omega} \times \underline{u}\right]=-\nabla p+\rho g \\
\nabla \cdot \underline{u}=0 \\
\left(\frac{\partial}{\partial t}+\underline{u} \cdot \nabla\right) \rho=0
\end{gathered}
$$

where $\rho, \underline{u}, p$ represent the density, fluid velocity, and the pressure respectively.

We study the problem under the assumption of the Rossby $\beta$-plane approximation, so that the Coriolis parameter $f=2 \Omega \sin \theta$ can be expanded in a Taylor series about a reference latitude $\theta_{0}$ to obtain

$$
f=2 \Omega \sin \theta=2 \Omega\left[\sin \theta_{0},\left(\theta-\theta_{0}\right) \cos \theta_{0}\right]=f_{0}+\beta y,
$$

with $f_{0}=2 \Omega \sin \theta_{0}, \quad \beta=\frac{\partial f}{\partial y}=\left(\frac{2 \Omega}{R}\right) \cos \theta_{0}$ at $\theta=\theta_{0}, y=R\left(\theta-\theta_{0}\right)$, and $R$ is the mean radius of the Earth.

With reference to the rectangular Cartisian coordinates $x$ (eastward), $y$ (northward) and $z$ (vertical), the basic equilibrium state is given by

$$
p=p_{0}, \rho=\rho_{0}, \underline{u}=(u, v, w)=(U(y), 0,0)
$$

where $p_{0}$ is the pressure, $\rho_{0}$ is the density and $U(y)$ is the basic velocity which depends on $y$ only. Substituting (2.5) into (2.1) - (2.3) with (2.4), we obtain

$$
\frac{\partial p_{0}}{\partial x}=0, \frac{\partial p_{0}}{\partial y}=-U f \rho_{0}, \frac{\partial p}{\partial z}=-\rho_{0} g
$$

We also assume that the basic density distribution varies with $z$ so that

$$
\frac{\partial}{\partial z}\left(\ln \rho_{0}\right)=-\sigma
$$

It follows from (2.6) that

$$
\begin{gathered}
\frac{1}{\rho_{0}} \frac{\partial \rho_{0}}{\partial y}=-\frac{1}{g} f U \sigma=-\alpha . \\
\frac{1}{\rho_{0}} \frac{\partial \rho_{0}}{\partial z}=-\sigma
\end{gathered}
$$

We next impose a small perturbation with respect to the equilibrium state, so that it induces changes in pressure, density, and velocity fields given by

$$
p=p_{0}+p^{*}, \rho=\rho_{0}+\rho^{*},(u, v, w)=\left(U+u^{*}, v^{*}, w^{*}\right),
$$


where $p^{*}, \rho^{*},\left(u^{*}, v^{*}, w^{*}\right)$ are the perturbation quantities.

Substituting these into $(2.1)-(2.3)$ and invoking linearization, we obtain the following equations:

$$
\begin{gathered}
\rho_{0}\left(\frac{\partial u^{*}}{\partial t}+U \frac{\partial u}{\partial x}+v^{*} \frac{\partial U}{\partial y}-v^{*} f\right)=-\frac{\partial p^{*}}{\partial x} \\
\rho_{0}\left(\frac{\partial v^{*}}{\partial t}+U \frac{\partial v^{*}}{\partial x}+u^{\star} f\right)+\rho^{\star} U f=-\frac{\partial p^{*}}{\partial y} \\
\rho_{0}\left(\frac{\partial w^{*}}{\partial t}+U \frac{\partial w^{*}}{\partial x}\right)=-\left(\frac{\partial p^{*}}{\partial z}+g \rho^{\star}\right) \\
\frac{\partial \rho^{*}}{\partial t}+U \frac{\partial \rho^{*}}{\partial x}+v^{\star} \frac{\partial \rho_{0}}{\partial y}+w^{*} \frac{\partial \rho_{0}}{\partial z}=0 \\
\frac{\partial u^{*}}{\partial x}+\frac{\partial v^{*}}{\partial y}+\frac{\partial w^{*}}{\partial z}=0
\end{gathered}
$$

where the coefficients of these equations are function of $y$ and $z$.

Using Eckart's transformations

$$
\left(p^{\star}, \rho^{\star}\right)=\rho_{0}^{\frac{1}{2}}(p, \rho),\left(u^{*}, v^{*}, w^{*}\right)=\rho_{0}^{-\frac{1}{2}}(u, v, w)
$$

the above system of equations reduces to one in which the coefficients are independent of $x, z$, and $t$. Therefore, it is then possible to assume normal mode solutions in the form

$$
\phi=\phi(y) \exp [i(k x+m z-\omega t)]
$$

We next eliminate all the dependent variables in favor of the velocity field $v$ to obtain a single equation for $v$ :

$$
\begin{aligned}
& \quad A \Omega_{d} \frac{d^{2} v}{d y^{2}}+\left(A^{\prime} \Omega_{d}-2 R i \Omega_{d}\right) \frac{d v}{d y}+\left[S^{\prime}+C+D U^{\prime}-i R^{\prime}-i B U^{\prime}\right] v=0 \\
& \text { where } \Omega_{d}=k U-\omega, A=Q^{-1}\left[\Omega_{d}^{2}-g \sigma\right], R=Q^{-1}[-\alpha g m] \\
& S=Q^{-1}\left[U^{\prime} g \sigma k+f k \Omega_{d}^{2}-f g \sigma k+\frac{\Omega_{d}^{3}}{2}-k U^{\prime} \Omega_{d}^{2}\right] \\
& Q=k^{2} N^{2}-\Omega_{d}^{2}\left(k^{2}+m^{2}+\sigma^{2} / 4\right), \alpha=\frac{f U_{\sigma}}{g}, B=Q^{-1}[U f k m \sigma] \\
& D=Q^{-1}\left[\frac{\alpha}{2} k \Omega_{d}^{2}-\frac{f \sigma^{2} \Omega_{d}^{2}}{4}-m^{2} f \Omega_{d}\right]
\end{aligned}
$$




$$
\begin{aligned}
C= & Q^{-1}\left[U \mathrm{fm}^{2} \Omega_{d} \alpha+U \mathrm{fk}^{2} \alpha \Omega_{d}+\frac{f^{2} \sigma^{2} \Omega_{d}}{2}+m^{2} f^{2} \Omega_{d}\right. \\
& \left.-k^{2} \Omega_{d}^{3}+N^{2} k^{2} \Omega_{d}-m^{2} \Omega_{d}^{3}-\frac{\sigma^{2} \Omega_{d}^{3}}{4}-\frac{\alpha^{2} \Omega_{d}^{3}}{4}\right]
\end{aligned}
$$

and primes denotes the differentiation with respect to $y$.

It is noted that equation (2.18) is singular at $\Omega_{d}=0, A=0$ and $Q=0$. of these singularities, wave absorption takes place only at the singularities of $A=0$, where $A$ is a quadratic expression in $\Omega_{d}$ and hence there exist two distinct critical levels. Hence, the propagation properties of the waves near these singularities are of special interest and will be discussed in the following sections.

\section{WAVE ACTION FLUX}

The upward transfer of wave energy per unit area at any level is the mean rate of working of pressure forces in the fluids above, $E=\overline{p v}$, where the over bar denotes an average over a horizontal wave length

$$
E=\overline{p v}=\frac{1}{4}\left[p^{*} v+p v^{*}\right]=\frac{1}{2} \operatorname{Re}\left(p v^{*}\right)
$$

where * denotes the complex conjugate. It can be shown that

$$
E=\frac{1}{2} \operatorname{Re}\left[i v^{*} A \Omega_{d} \frac{d v}{d y}+(R+i S) v v^{*} \Omega_{d}\right]
$$

Now, we define $M$ as the wave action flux. In this case $M$ is proportional to the angular momentum flux about the rotation axis, as the ratio of wave energy flux to the local relative frequency, we obtain

$$
M=\frac{E}{\Omega_{d}}=\frac{1}{2} \operatorname{Re}\left[i v^{\star} A \frac{d v}{d y}+\operatorname{Rvv}^{\star}\right]
$$

Differentiating (3.3) with respect to $y$ and substituting the wave equation (2.18) into the resulting expression, we find, $\frac{d M}{d y}=0$ except at the critical levels, where substitution of (2.18) is not valid. Thus, $M$ is constant between critical levels, but it has a discontinuity at each critical level. In the absence of rotation, $M$ is proportional to the vertical flux of horizontal momentum, but in the presence of rotation, about an axis, $M$ may be related to the angular momentum flux about that axis, and conveniently acts as a measure of the intensity of the wave.

4. SINGULARITIES OF THE WAVE EQUATION AND BEHAVIOR OF ITS SOLUTION

(a) Singularity at $\Omega_{d}=N$ where $N$ is the Brunt Vaisala frequency.

It is noted that the wave equation has a regular singularity at $y_{c_{+}}$where

$$
\Omega_{d}\left(y_{c_{+}}\right)=k \cup\left(y_{c_{+}}\right)-\omega=N
$$


The method of Frobenius shows that $v(y)$ can be written as

$$
v(y)=\left(y-y_{c}\right)^{\lambda}\left[1+a_{1}\left(y-y_{c_{+}}\right)+\ldots\right]
$$

Substitution of (4.2) into (2.18) gives the indical equation for $\lambda$ which has solutions, $\lambda=0,+i \mu$ where $\mu=\left[2 R\left(y_{C_{+}}\right) / A^{\prime}\left(y_{c_{+}}\right)\right]$.

Hence,

$$
v=c_{1}\left[1+c_{11}\left(y-y_{c}\right)+\ldots\right]+c_{2}\left(y-y_{c}\right)^{i \mu}\left[1+c_{21}\left(y-y_{c}\right)+\ldots\right]
$$

(i) $\lambda=i_{\mu}$ : To determine the appropriate branch for $\left(y-y_{c_{+}}\right)^{\lambda}$ in (4.2), we follow Booker and Bretherton [1967]; it will be assumed that $(d A / d y)>0$, with $\omega_{i}>0$ $\left(\omega=\omega_{r}+i \omega_{j}\right)$, so that the amplitude of the wave at any section is slowly growing with time. Thus, $\ln \left(y_{c_{+}}\right)>$or $<0$ according as $\mu>0$ or $<0$. In the limit $\omega_{i} \rightarrow 0$, the appropriate path for determining the branch of $\left(y-y_{c_{+}}\right)$passes below (above) $y_{c_{+}}$

$$
\left(y-y_{c}\right)^{i \mu}=\begin{array}{lll}
\left|y-y_{c}\right|^{i \mu} \quad \text { for } & y>y_{c_{+}} \\
\left|y-y_{c}\right|^{i \mu} \exp (\mu \pi) \text { for } y<y_{c_{+}}
\end{array} \mid
$$

Substituting (4.4ab) into (4.2) we obtain

$$
\left.v=\begin{array}{ll}
c_{2}\left|y-y_{c}\right|^{i \mu}\left[1+c_{21}\left(y-y_{c}\right)+\ldots\right] & \text { for } y>y_{c_{+}} \\
c_{2}\left|y-y_{c}\right|^{i \mu} \exp (\mu \pi)\left[1+c_{21}\left(y-y_{c}\right)+\ldots\right] & \text { for } y<y_{c_{+}}
\end{array}\right\}
$$

Substituting the above expression for $v$ into (3.3) we obtain

$$
\left.M=\begin{array}{ll}
\frac{1}{2} R\left|C_{2}\right|^{2} & \text { for } y>y_{c_{+}} \\
\frac{1}{2} R\left|C_{2}\right|^{2} \exp (2 \mu \pi) & \text { for } y<y_{c_{+}}
\end{array}\right\}
$$

The energy

$$
E=\begin{array}{ll}
\frac{1}{2} R \Omega_{d}\left|C_{2}\right|^{2} & \text { for } y>y_{c_{+}} \\
\frac{1}{2} R \Omega_{d}\left|C_{2}\right|^{2} \exp (2 \mu \pi) & \text { for } y<y_{c_{+}}
\end{array} \mid
$$

Since the sign of $E=M \Omega_{d}$, determines the direction of wave, it follows that if $\mu \frac{d A}{d y}<(>0)$, the solution represents a wave propagating downwards (upwards). In either case the wave energy flux across the critical level is decreased by the factor $\exp (2 \mu \pi)$. The solution is characterised by the condition 


$$
v_{g} \Omega_{d} \frac{d A}{d y}<0
$$

where $V_{g}$ is the horizontal propagation velocity. (ii) $\lambda=0$ : This solution is regular at $y_{c_{+}}$. Evaluating (3.3) at $y=y_{c_{+}}$, we
obtain

$$
E=-\frac{1}{2} R \Omega_{d}\left|C_{1}\right|^{2}
$$

Hence, this solution represents a wave propagating in a direction opposite to the one we have just investigated. This solution is characterized by

$$
V_{g}^{\mu} \Omega_{d} \frac{d A}{d y}>0
$$

Thus, the critical level, $\Omega_{d}=N$ acts as a valve. A wave propagating towards a valve will be transmitted (or) unattenuated by a factor $\exp (2 \mu \pi)$ according as

$$
v_{g^{\mu}} \Omega_{d} \frac{d A}{d y}>\text { or }<0
$$

(b) Singularity at $\Omega_{d}=-N$.

There is a singularity at $y_{c_{-}}$where $\Omega_{d}\left(y_{c_{-}}\right)=-N$. Seeking a solution of the form (4.2) in the variables $\left(y-y_{c_{-}}\right)$leads to an indical equation for $\lambda$, with solutions $\lambda=0,-i \mu$.

An analysis similar to the above shows that for the solution given by $\lambda=-i \mu$ :

$$
E=\begin{array}{ll}
\frac{1}{2} R \Omega_{d}\left|C_{2}\right|^{2} & \text { for } y>y_{c_{-}} \\
\frac{1}{2} R \Omega_{d}\left|C_{2}\right|^{2} \exp (-2 \mu \pi) & \text { for } y<y_{c_{-}}
\end{array} \mid
$$

Hence, this solution represents a wave propagating vertically downwards (or) upwards according as $\mu \frac{d A}{d y}>$ or $<0$. In either case, the wave energy flux is attenuated by the factor $\exp (2 \mu \pi)$.

This solution is again characterised by

$$
v_{g} \mu \Omega_{d} \frac{d A}{d y}>0
$$

For the solution given by $\lambda=0$

$$
E=-\frac{1}{2} R \Omega_{d}\left|C_{1}\right|^{2}
$$

and hence represents a wave propagating downwards (or) upwards according as $\mu \frac{d A}{d y}>$ or $<0$, the solution is characterized by

$$
v_{g} \mu \Omega_{d} \frac{d A}{d y}<0
$$

Thus, the critical level $\Omega_{d}=-N$ also acts as a valve, operating on the same criterion as the critical level at $\Omega_{d}=N$. A wave propagating towards a critical level is transmitted (or) absorbed according as

$$
v_{g} \mu \Omega_{d} \frac{d A}{d y}<\text { or }>0
$$


(c) Singularity at $\Omega_{d}=0$.

There is a regular singularity at $y_{0}$, where $k U\left(y_{0}\right)=\omega$. Seeking a solution of the form (4.2) in the variables $y-y_{0}$ leads to an indical equation for $\lambda$, with solutions, $\lambda=0,1$. The general solution is thus regular and has the form

$$
V(y)=a_{0}\left[1+a_{01}\left(y-y_{0}\right)+\ldots\right]
$$

where $a_{0}$ is an arbitrary constant and $a_{01}, \ldots$ are known constants. Substitution of (4.17) into (3.3) shows that $M$ is a regular function of $y$ near $y_{0}$.

A similar solution and conclusion hold near the singularity at $Q=0$.

\section{WAVE PROPAGATION IN A SLOWLY VARYING MEDIUM}

When the properties of the medium vary only slightly over distance of the order of a wavelength, the concept of a wavegroup is extremely helpful.

If $U(y)$ and $N^{\prime}$ do not vary much over a wavelength, the wave equation with horizontal wave numbers $k$ and $\ell$ and vertical wave number $m$ satisfies the dispersion relation

$$
\begin{aligned}
& l_{d}^{2}\left[\Omega^{3}-N_{d}^{2} \Omega\right]+2 \operatorname{loUfm} \Omega_{d}+\Omega_{d}^{3}\left[k^{2}+m^{2}+\frac{\alpha^{2}+\sigma^{2}}{4}\right] \\
& -\left[N^{2} k^{2}+m^{2} f^{2}+\frac{\sigma^{2} f^{2}}{2}+U f k_{\alpha}^{2}+U f m_{\alpha}^{2}\right] \Omega_{d}+g \sigma k \beta=0
\end{aligned}
$$

If $k, \omega, \ell$ and $m$ vary with position and time and $U$ with $y$ only, we may write the above dispersion relation in the form

$$
\Omega_{d}=D(k, l, m, y)
$$

and then formally define the group velocity

$$
\underline{U}_{g}=\left(U_{g}, V_{g}, W_{g}\right)=\left(\frac{\partial D}{\partial k}, \frac{\partial D}{\partial l}, \frac{\partial D}{\partial m}\right)
$$

which therefore also varies with position and time. The significance of the group velocity as defined above is that an observer moving always with the local group velocity will find locally a constant value of the horizontal wave numbers and frequency, however, since the background velocity varies horizontally, will change in horizontal wave number $\ell$. Thus, the role of (5.1) when the background velocity is not uniform is (i) to enable us to calculate the horizontal wave number at each level in terms of the local background velocity at that level, having selected a particular group (that is, having assigned values $\omega, k$ and $m$ and then ( $i i$ ) to permit us to use these values to calculate the group velocity at every level and in this way compute the trajectory of the group. We now use this procedure to study the propagation of wave groups in the neighborhood of the critical level. 
As the group approaches its critical level $y=y_{c}$, at which

$$
\left[\Omega_{d}^{2}-N^{2}\right]_{y_{c}}=0
$$

one group of (5.1) increases indefinitely and is given asymptotically by

$$
\left[\Omega_{d}^{2}-N^{2}\right] \ell \simeq-2 \sigma m U f
$$

The asymptotic behavior of the horizontal component of the group velocity $V_{g}$ corresponding to the root of $(5.5)$ is given by

$$
\frac{\partial \omega}{\partial l}=V_{g}=\frac{\text { omUf }}{\Omega_{d} l_{\infty}^{2}} .
$$

Using (5.4) and (5.5), we obtain

$$
v_{g}=\frac{1}{4 \sigma m U f \Omega_{d}}\left(\frac{d A}{d y}\right)^{2}\left(y-y_{c}\right)^{2}
$$

Thus, when $\left(y-y_{c}\right)$ is small, the distance $y$ of the group satisfies

$$
\frac{d y}{d t}=a\left(y-y_{c}\right)^{2}
$$

where $a$ is a constant. On integrating the equation we obtain

$$
\left(y-y_{c}\right)=-1 /(a t+b)
$$

Hence, it follows that the time taken by a group to pass from a level $y_{1}$ to level $y_{2}$ is

$$
t_{2}-t_{1}=\frac{1}{a}\left[\frac{1}{y_{2}-y_{c}}-\frac{1}{y_{1}-y_{c}}\right]
$$

which is arbitrarily large if $y_{2}$ is sufficiently near $y_{c}$. In other words, the wavegroup takes an infinite time to reach the critical level. So, the group is neither transmitted nor reflected and simply slows down until either diffusion, turbulence, or other non-linearities destroy it.

This group is characterized by

$$
v_{g} \text { omUf } \Omega_{d}>0
$$

The other root of (5.1) tends to a finite value $\ell_{f}$ as the critical level is approached, and accordingly $v_{g}$ tends to a non-zero finite value there, and the group according to $\ell_{f}$ is transmitted across the critical level. This group approaches the critical level from a direction such that

$$
V_{g} \text { omUf } \Omega_{d}<0
$$

Thus, a wave approaching its critical level is either captured or transmitted according as

$$
V_{g} \text { omUf } \Omega_{d}>\text { or }<0
$$


The picture presented in this section, with wavenumbers varying only very slightly over distances of the order of a wavelength and complete capture rather than partial transmission, arises naturally out of the picture presented in the previous section when $|\mu|>1$ (formally, as $\mu \longrightarrow \infty$ ).

\section{DISCUSSIONS AND CONCLUSIONS}

The wave equation is derived for Rossby waves in an isothermal atmosphere on a beta plane in the presence of a latitudinally sheared zonal flow. This equation is singular at $\Omega_{d}= \pm N, \Omega_{d}=0$ and $Q=0$. The first two singularities are different in form from the singularities obtained in the case of a vertically rotating stratified atmosphere. The wave action flux which is the ratio of the wave energy flux to the local relative frequency (which is proportional to the angular momentum flux) is constant everywhere inside the fluid except at the critical level; we find it as an appropriate measure of the magnitude of the waves when the propagation of waves across the critical level is studied. Using this wave action flux, it is shown that the waves are transmitted across the critical level $\Omega_{d}=0, Q=0$ without any attenuation, while the other two critical levels $\pm N$ behave as valves by permitting waves to penetrate them without any attenuation one way only.

Also at these critical levels, an absorption coefficient $\exp (2 \mu \pi)$ is obtained. In this way, we have used Rossby wave problem as an example to demonstrate that the normal mode solution with critical level behavior in some cases bears no resemblance to the initial value problem, not even in the infinite time limit.

ACKNOWLEDGEMENT. This research was partially supported by the University of Central Florida.

\section{REFERENCES}

1. LONGUET-HIgGiNS, M. S. Planetary Waves on a Rotating Sphere, Proc. Roy. Soc. London, A 278 (1964) 446-473.

2. LONGUET-HIGgINS, M. S. Planetary waves on a Rotating Sphere II, Proc. Roy. Soc. London, A 279 (1965) 40-68.

3. DEBNATH. L., On Wind-Driven Currents in an Ocean with Bottom Friction, Z. Angew Math and Mech. 56 (1976), 469-478.

4. CHARNEY, J. G., and DRAZIN, P. G., Propagation of Planetary-Scale Disturbances from the Lower into the Upper Atmosphere, J. Geophys. Res. 66 (1961), 83-99.

5. DICKINSON, R. E. Planetary Rossby Waves Propagating vertically Through Weak Westerly Wind Wave Guides, J. Atmos. Sci. 25 (1968) 984-994.

6. LINDZEN, R. S., Internal Equitorial Planetary-Scale Waves in a Shear Flow, $\underline{\mathrm{J}}$. Atmos. Sci. 27 (1970) 394-405. 
7. BOOKER, J. R. and BRETHERTON, F. P., The Critical Layer for Internal Gravity Waves in a Shear Flow, J. Fluid Mech. 27 (1967) 513-539.

8. MEKKI, 0. M., and McKENZIE, J. F., The Propagation of Atmospheric Rossby Gravity Waves in Latitudinally Sheared Zonal Flows, Phil. Trans. Roy. Soc. London A287 (1977) $115-143$.

9. GREENSPAN, H. P., The Theory of Rotating Fluids, Cambridge University Press, 1966 . 


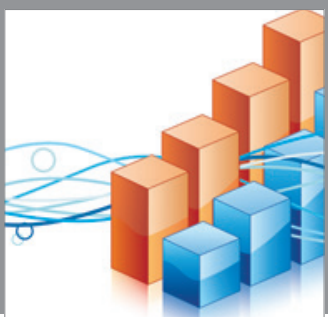

Advances in

Operations Research

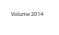

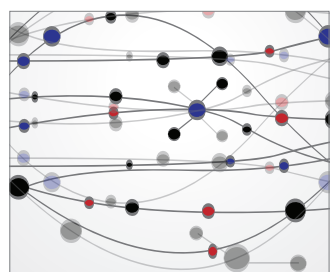

\section{The Scientific} World Journal
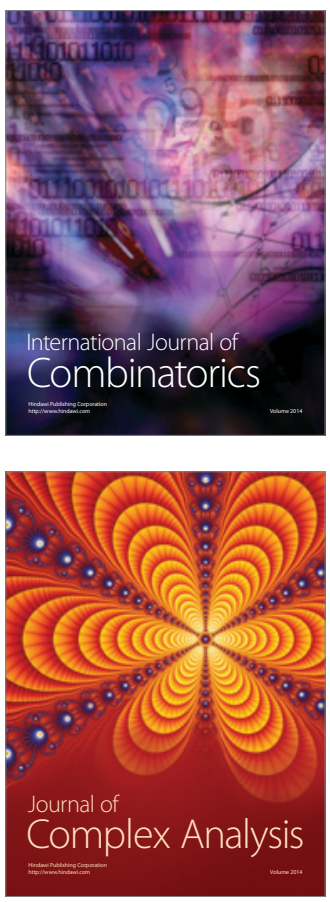

International Journal of

Mathematics and

Mathematical

Sciences
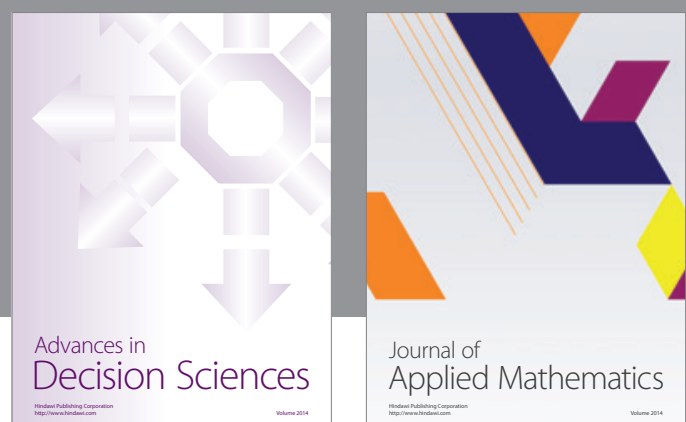

Journal of

Applied Mathematics
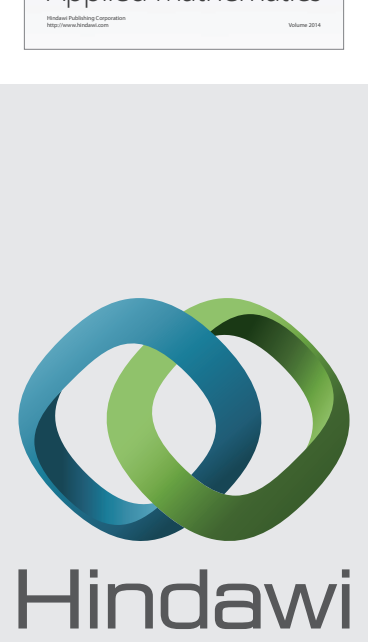

Submit your manuscripts at http://www.hindawi.com
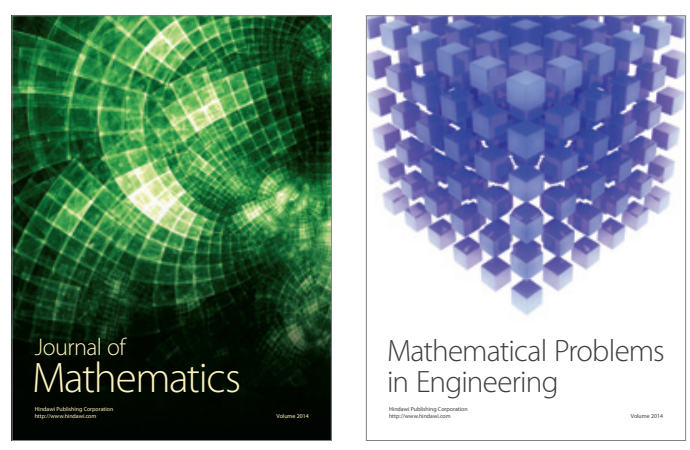

Mathematical Problems in Engineering
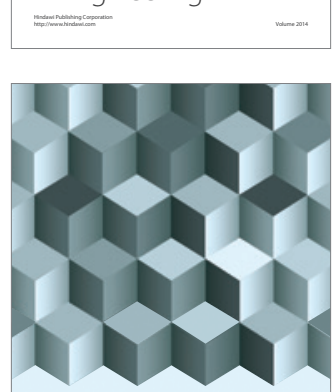

Journal of

Function Spaces
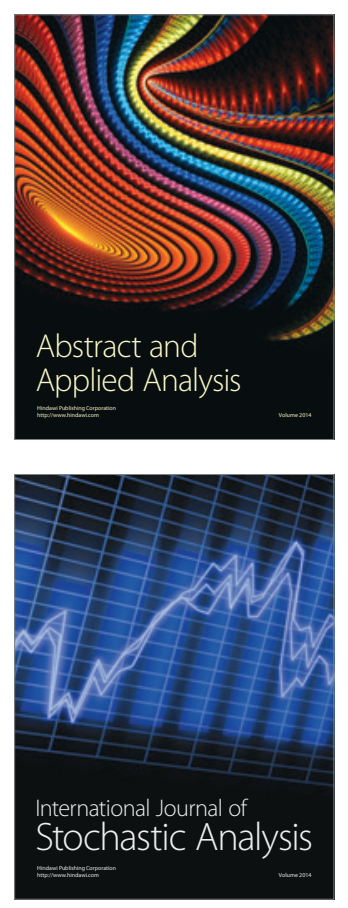

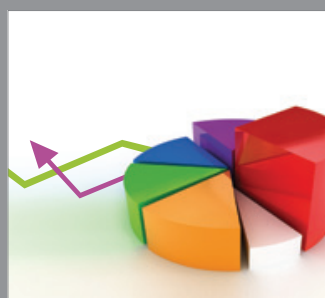

ournal of

Probability and Statistics

Promensencen
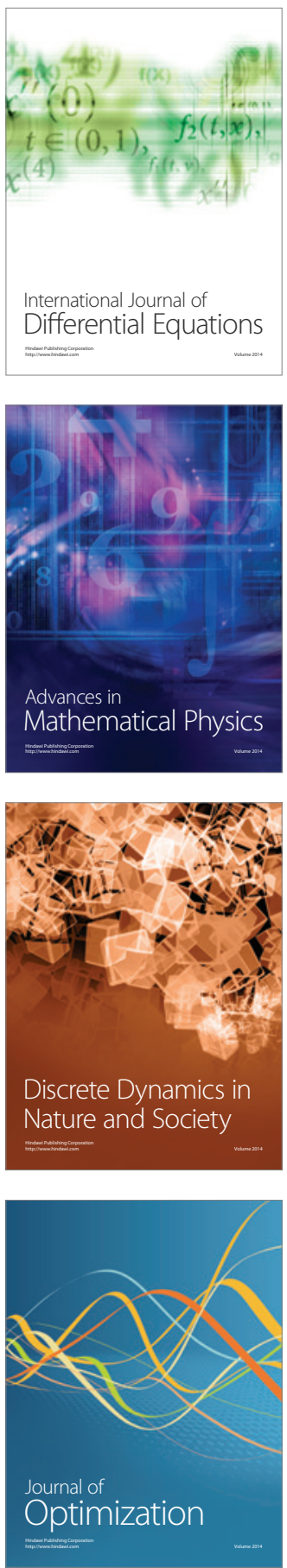